\title{
News and Views on STAT3 Psychopathology
}

\author{
Sara H. Sadok ${ }^{1}$, Rayssa L. Borges-Medeiros ${ }^{1}$, Paula V. B. Macêdo ${ }^{1}$, \\ João Ricardo M. de Oliveira ${ }^{1,2 *}$ and High IgE Consortium \\ ${ }^{1}$ Keizo Asami Laboratory, Federal University of Pernambuco, Recife, Brazil, ${ }^{2}$ Neuropsychiatric Department, Federal University of \\ Pernambuco, Recife, Brazil
}

Keywords: Job's syndrome, high IgE, signal transducer and activator of transcription, neuropsychiatric disorders, biomarker

\section{INTRODUCTION}

The work of Reisinger and others from Austria, despite being based on experiments conducted on rodents, claims an old ambition of neuroimmunologists, namely, to integrate the main psychopathology disturbances into a unified paradigm (Reisinger et al., 2020). These authors published in the Molecular Psychiatry Journal a study concerning the signal transducer and activator of transcription 3 protein (STAT3) and its regulation of emotional behavior in knockout and knockdown Stat3 mice (Reisinger et al., 2020). They highlighted the potential role played by Stat 3 protein in the serotonergic system and, therefore, suggested the implications in the genesis of neuropsychiatric disorders, reinforcing the well-known immune hypothesis of mental illness.

By analyzing the achieved results, it is essential to rethink the current paradigm of human neuropsychiatry disorders concerning diagnosis and consider that biological markers might be a breakthrough. However, the main challenge still is to tackle the first domino piece on the neuroimmune cascade leading to sustained changes triggered by environment distress of even "primary" or "endogenous" and sometimes even predictable swings on mood, attention, memory and full vigilance. Curiously, the neuroimmune concept is being explored for over a century and we remember the 1927 Nobel Winner, Julius Wagner-Jauregg, also from Austria, and his work using many microbes to treat mainly psychotic and catatonic patients.

\section{DISCUSSION}

It is important to point that current database, such as the Allen Brain Atlas $\left({ }^{\odot} 2008\right.$ Allen Institute for Brain Science. Allen Developing Mouse Brain Atlas. Available from: https://developingmouse.brainmap.org/, and ${ }^{\odot} 2004$ Allen Institute for Brain Science Allen Mouse Brain Atlas. Available from: https://mouse.brain-map.org/) and the mouse brain vasculature map provided by Betsholtz Lab database (Vanlandewijck et al., 2018), in wild-type mice shows that Stat3 is widely expressed in different cell types, showing peaks of expression in arterial smooth muscle cells (aSMC) and subtype 2 vascular fibroblast-like cells (Fb2), and a lower level at astrocytes (AC). In the developing mouse brain, Stat 3 has a similar expression pattern in all areas. However, there is a change in the expression pattern between the embryonic days and postnatal age in the developing mouse brain, since during the postnatal days (P4, P14 and P28) the Stat3 expression values increase. In the adult mouse brain, most areas also share a similar expression profile of this gene.

In the context of the classical monoamine hypothesis, for most part of the time cerebrospinal fluid levels of serotonin, dopamine and noradrenaline and their respective enzymes, as well as other neurotransmitters, have been considered as the main biomarkers of mental illnesses, especially in 
major depressive disorders. Recently, studies have also suggested that neurotrophic and inflammatory biomarkers may play a role in the pathogenesis of these psychiatric disorders. There is increased evidence that elevated levels of cytokines, C-reactive protein and interleukins are related to the pathogenesis of psychiatric conditions and hence could be used as measurable indicators in the scenario of mental disorders (Lozupone et al., 2019). Moreover, the potential use of biomarkers is not limited to aid with precision regarding diagnostic criteria, but also related to response to treatment, stratification of patients and appropriate treatment choice in the era of Personalized Medicine (GarciaGutiérrez et al., 2020).

In humans, STAT3 gene variants, associated with both autosomal dominant and sporadic form are linked to the Hyper Immunoglobulin E (Hyper-IgE) syndrome (also known as Job's disease). The Hyper-IgE syndrome is a group of different inborn errors of immunity marked by the presence of recurrent infections, multisystem organs involvement, characteristic facial dysmorphism, dermatitis, vasculopathy, and extremely elevated serum levels of IgE (Tsilifis et al., 2021). Although STAT3 variants in animal models were involved in behavioral implications, also mentioned by Reisinger et al., psychiatric symptoms were not listed in the clinical description of the referred syndrome.

It has come to our attention that similar, but mild, symptomatology of this syndrome might be present in some patients with mildly increased IgE serum levels, which remains not documented in the literature (Sadok et al., 2021). As a consequence, these changes might result in a broad range of clinical presentations, involving moderate symptoms and including neuropsychiatric disorders. The referred manifestations are usually considered benign and common in the population, depending on the type of complication and the system affected. In addition, they are intensified on consanguineous families (Otto et al., 2020).

\section{REFERENCES}

García-Gutiérrez, M. S., Navarrete, F., Sala, F., Gasparyan, A., Austrich-Olivares, A., and Manzanares, J. (2020). Biomarkers in Psychiatry: Concept, Definition, Types and Relevance to the Clinical Reality. Front. Psychiatry. 11, 432. doi:10.3389/ fpsyt.2020.00432

Lozupone, M., La Montagna, M., D’Urso, F., Daniele, A., Greco, A., Seripa, D., et al. (2019). The Role of Biomarkers in Psychiatry. Adv. Exp. Med. Biol. 1118, 135-162. doi:10.1007/978-3-030-05542-4_7

Otto, P. A., Lemes, R. B., Farias, A. A., Weller, M., Lima, S. O. A., Albino, V. A., et al. (2020). The Structure of First-Cousin Marriages in Brazil. Sci. Rep. 10 (1), 15573. doi:10.1038/s41598-020-72366-Z

Reisinger, S. N., Sideromenos, S., Horvath, O., Derdak, S., Cicvaric, A., Monje, F. J., et al. (2020). STAT3 in the Dorsal Raphe Gates Behavioural Reactivity and Regulates Gene Networks Associated With Psychopathology. Mol. Psychiatry. 26, 2886-2899. doi:10.1038/s41380-020-00904-2

Sadok, S. H., Borges-Medeiros, R. L., and de Oliveira, J. R. M. (2021). Editorial of Concern. J. Mol. Neurosci., 1. doi:10.1007/s12031-021-01904-9

Troyer, E. A., Kohn, J. N., and Hong, S. (2020). Are We Facing a Crashing Wave of Neuropsychiatric Sequelae of COVID-19? Neuropsychiatric Symptoms and Potential Immunologic Mechanisms. Brain Behav. Immun. 87, 34-39. doi:10.1016/j.bbi.2020.04.027

Tsilifis, C., Freeman, A. F., and Gennery, A. R. (2021). STAT3 Hyper-IgE Syndrome-An Update and Unanswered Questions. J. Clin. Immunol. 41 (5), 864-880. doi:10.1007/s10875-021-01051-1
During the COVID-19 pandemic, the neurological implications of the infection and the affinity of the virus for the brain grabbed the attention of the scientific community. In addition, the relation between neuropsychiatric symptoms development on COVID-19 patients and increased pro-inflammatory cytokines response to the virus infection has been demonstrated (Varatharaj et al., 2020). Therefore, this information reinforces the relevance of biomarkers screening for risk stratification or even diagnosis. Interestingly, Troyer et al. (2020) detected on fatal COVID-19 patients, increased pro-inflammatory interleukin 6 , one of the triggers for STAT3 pathway activation.

\section{CONCLUSION}

Thus, we believe that the findings described by the authors in the manuscript, along with our hypothesis of neuropsychiatric involvement of STAT3 Hyper-IgE patients and the importance of biomarkers, should be considered in future research.

\section{AUTHOR CONTRIBUTIONS}

SS, RB-M, PM, and JO drafted the manuscript. PM was responsible for Allen Brain Atlas analysis. JO conceived and designed the study. JO provided final approval of the version to be submitted. The manuscript was reviewed and approved by all authors.

\section{FUNDING}

This work was supported by CNPq (Grant \#311664/2020-2), FACEPE (Grand \#IBPG-0749-2.02/16) and PROPESQI-UFPE.

Vanlandewijck, M., He, L., Mäe, M. A., Andrae, J., Ando, K., Del Gaudio, F., et al. (2018). A Molecular Atlas of Cell Types and Zonation in the Brain Vasculature. Nature. 554, 475-480. doi:10.1038/nature25739

Varatharaj, A., Thomas, N., Ellul, M. A., Davies, N. W. S., Pollak, T. A., Tenorio, E. L., et al. (2020). Neurological and Neuropsychiatric Complications of COVID-19 in 153 Patients: a UK-Wide Surveillance Study. Lancet Psychiatry. 7 (10), 875-882. doi:10.1016/S2215-0366(20) 30287-X

Conflict of Interest: The authors declare that the research was conducted in the absence of any commercial or financial relationships that could be construed as a potential conflict of interest.

Publisher's Note: All claims expressed in this article are solely those of the authors and do not necessarily represent those of their affiliated organizations, or those of the publisher, the editors and the reviewers. Any product that may be evaluated in this article, or claim that may be made by its manufacturer, is not guaranteed or endorsed by the publisher.

Copyright (C) 2021 Sadok, Borges-Medeiros, Macêdo, de Oliveira and High IgE Consortium. This is an open-access article distributed under the terms of the Creative Commons Attribution License (CC BY). The use, distribution or reproduction in other forums is permitted, provided the original author(s) and the copyright owner(s) are credited and that the original publication in this journal is cited, in accordance with accepted academic practice. No use, distribution or reproduction is permitted which does not comply with these terms. 